\title{
Dimensionality of the spatiotemporal entanglement of parametric down-conversion photon pairs
}

\author{
A. Gatti, ${ }^{1,2}$ T. Corti, ${ }^{2}$ E. Brambilla, ${ }^{2}$ and D. B. Horoshko ${ }^{3,4}$ \\ ${ }^{1}$ Istituto di Fotonica e Nanotecnologie del CNR, Piazza Le Da Vinci 32, Milano, Italy \\ ${ }^{2}$ Dipartimento di Scienza e Alta Tecnologia dell' Università dell'Insubria, Via Valleggio 11, Como, Italy \\ ${ }^{3}$ Laboratoire PhLAM, Université de Lille 1, 59655 Villeneuve d'Ascq, France \\ ${ }^{4}$ B. I. Stepanov Institute of Physics, NASB, Nezavisimosti Avenue 68, Minsk 220072 Belarus
}

(Received 25 July 2012; published 5 November 2012)

\begin{abstract}
In this work, the Schmidt number of the two-photon state generated by parametric down-conversion (PDC) is evaluated in the framework of a fully spatiotemporal model for PDC. A comparison with the results obtained in either purely spatial or purely temporal models shows that the degree of entanglement of the PDC state cannot be trivially reduced to the product of the Schmidt numbers obtained in models with lower dimensionality, unless the detected bandwidth is very narrow. This result is a consequence of the nonfactorability of the state in the spatial and temporal degrees of freedoms of twin photons. In the limit of a broad pump beam, we provide a geometrical interpretation of the Schmidt number as the ratio between the volume of the phase-matching region and of a correlation volume.
\end{abstract}

DOI: 10.1103/PhysRevA.86.053803

PACS number(s): 42.65.Lm, 42.50.Dv, 03.65.Ud

\section{INTRODUCTION}

The process of parametric down-conversion (PDC) occurring in a nonlinear crystal is a widely employed source of entangled photons, which are ubiquitous ingredients in modern quantum technologies. An appealing aspect of this source is the possibility of generating high-dimensional entanglement, both in the sense that entanglement is generated in various degrees of freedom of the photon pair (polarization, timeenergy, position-momentum) and because spatial and temporal entanglement is realized in a high-dimensional Hilbert space, due to the naturally ultrabroad bandwidths of the process. High-dimensional entanglement is attracting more and more attention because of its potential to increase the capacity and the security of quantum communication channels, and the precision of quantum metrological techniques [1].

In this context, an obvious question concerns the effective dimensionality of the entanglement of the PDC state (or, alternatively, the number of entangled modes generated by the process), which is usually quantified by the so-called Schmidt number $[2,3]$. Traditional approaches typically concentrate on a single degree of freedom at a time, depending on the application considered. For example, the dimensionality of the temporal entanglement has been evaluated in various configurations, including spontaneous PDC [4,5], quantum frequency combs generated by a synchronously pumped optical parametric oscillator [6,7], and waveguided PDC [8]. The degree of transverse spatial entanglement of PDC [9-13] is of paramount importance for assessing both the dimensionality of the orbital angular momentum entanglement (see, e.g. Refs. [11,12,14-17]) and the resolution of quantum imaging techniques [18]. In these studies, a net separation between the spatial and temporal degrees of freedom was often justified by the assumption of a narrow filtering in the neglected degree of freedom.

However, as for many nonlinear optical processes, PDC is ruled by phase matching, which imposes an angular dispersion relation linking the frequencies and the angles of emission of the generated photons in a nonfactorable way. This implies a strong coupling between temporal and spatial degrees of freedom, recently evidenced by the so-called $X$ entanglement [19-24], which is a feature shown, for example, by type-I biphotons in conditions close to collinear phase matching, whose temporal delays and transverse spatial displacements at the crystal exit face are linked by a proportionality relation (corresponding to an $\mathrm{X}$ shape of the spatiotemporal correlation in any plane containing time and one transverse coordinate). The space-time coupling is often regarded as a negative feature because it affects, e.g., the purity of the purely spatial entanglement when temporal degrees of freedom are neglected [25]. However, it also represents a valuable resource for engineering the quantum state of biphotons, since the spatial degrees of freedom can be used to tailor the temporal entanglement $[19,20]$ in order to realize, e.g., an ultrabroadband temporally entangled state [22].

In this work, we adopt a fully spatiotemporal model of PDC in order to investigate the effect of the nonfactorability of the state in space and time on the dimensionality of the biphoton entanglement. We shall concentrate on the evaluation of the spatiotemporal Schmidt number of PDC entanglement in the ultralow gain regime of PDC. ${ }^{1}$ Based on a general formula for the Schmidt number, which involves integrals in 12 and six dimensions, we obtain both numerical evaluations and analytical results, with the latter being valid when the profile of the pump driving the process is broad enough. In the same limit, we introduce a useful geometric interpretation of our results, which shows that the Schmidt number quantifying entanglement is basically the ratio between the volume of the region where phase matching efficiently occurs and a correlation volume, thus being proportional to the number of spatiotemporal correlated modes.

\footnotetext{
${ }^{1}$ The Schmidt decomposition of the state in the full spatiotemporal domain is very hard to achieve (even numerically) due to the hyperbolic geometry of the phase-matching relations. However, the Schmidt number can be computed even without performing the decomposition.
} 
The same methods of analysis are used to calculate the Schmidt number in a purely two-dimensional (2D) spatial model and in a purely 1D temporal model of PDC. An important result that will be demonstrated is that the Schmidt dimensionality of the 3D model of PDC cannot be trivially reduced to the product of the Schmidt numbers in the models of lower dimensionality. As a matter of fact, the Schmidt dimensionality of the full PDC state is by far larger than what would be expected from an approach that considers space and time as separable degrees of freedom, showing that an enormous number of entangled modes is available for the down-converted light. This result is again a consequence of the intrinsic nonfactorability of the state in its spatial and temporal degrees of freedom, and shows that using a full spatiotemporal model for describing PDC is essential in order to correctly quantify the degree of entanglement of the state.

The results presented in this work extend and complement those presented in Ref. [26], where a 2D spatiotemporal model (one temporal dimension + one spatial dimension) for PDC has been investigated.

\section{STARTING POINT}

In Refs. [19,20,27], the spatiotemporal quantum properties of the PDC light were described by studying the evolution of the quantum field operators through the nonlinear crystal, and deriving input-output relations linking the operators at the crystal output face with those at the entrance face. Here we consider the equivalent state formalism, in which the state evolves from the input to the output face of the crystal. We focus on type-I PDC in the regime of ultralow gain where the probability of generating a photon pair in each spatiotemporal mode is small (more precisely, the probability of generating more than one photon pair in each mode is negligible). The output biphoton quantum state can thus be written as a generic superposition of the vacuum state $|0\rangle$ and of a state with two photons generated in all possible spatiotemporal modes:

$\left|\psi_{\mathrm{PDC}}\right\rangle=|0\rangle+\int d \vec{w}_{1} \int d \vec{w}_{2} \mathcal{C}\left(\vec{w}_{1}, \vec{w}_{2}\right) A^{\dagger}\left(\vec{w}_{1}\right) A^{\dagger}\left(\vec{w}_{2}\right)|0\rangle$,

where $A$ is the quantum field operators for the down-converted field, and $\vec{w}$ indicates the full $3 \mathrm{D}$ spatiotemporal Fourier coordinate with the shorthand notation

$$
\vec{w}=(\vec{q}, \Omega),
$$

where $\vec{q}$ is the transverse component of the photonic wave vector $\vec{q}=\left(q_{x}, q_{y}\right)$ with respect to the mean propagation direction $z$ of the pump field, and $\Omega=\omega-\omega_{p} / 2$ is the temporal frequency shift from the central frequency of the PDC emission. The coordinate in the direct transverse space-time domain will be denoted by

$$
\vec{\xi}=(\vec{x}, t),
$$

where $\vec{x}=(x, y)$ is the spatial coordinate spanning the transverse plane at the crystal exit face and $t$ is time, with the convention

$$
\vec{w} \cdot \vec{\xi}=\vec{q} \cdot \vec{\xi}-\Omega t
$$

The term $\mathcal{C}\left(\vec{w}_{1}, \vec{w}_{2}\right)$ in Eq. (1) is the probability amplitude of generating a photon pair in the spatiotemporal modes $\vec{w}_{1}$ and $\vec{w}_{2}$, and can be determined by exploiting the equivalence with the field formalism developed in Refs. [19,20,27]. In these references, the biphoton amplitude was calculated in terms of the field-field correlation at the crystal output face,

$$
\begin{aligned}
\psi\left(\vec{w}_{1}, \vec{w}_{2}\right) & =\left\langle A\left(\vec{w}_{1}, l_{c}\right) A\left(\vec{w}_{2}, l_{c}\right)\right\rangle \\
& =\frac{g}{(2 \pi)^{\frac{3}{2}}} \tilde{\mathcal{A}}_{p}\left(\vec{w}_{1}+\vec{w}_{2}\right) \operatorname{sinc} \frac{\Delta\left(\vec{w}_{1}, \vec{w}_{2}\right)}{2} e^{i \frac{\Delta\left(\vec{w}_{1}, \vec{w}_{2}\right)}{2}},
\end{aligned}
$$

where $A\left(\vec{w}, l_{c}\right)$ is the output field operator, and the expectation in Eq. (5) is taken on the input vacuum state; $g$ is the dimensionless gain parameter, proportional to the pump peak amplitude, the crystal length, and the nonlinear susceptibility; $\tilde{\mathcal{A}}_{p}$ is the Fourier transform of the pump beam profile at the crystal exit face,

$$
\tilde{\mathcal{A}}_{p}(\vec{w}):=\int \frac{d \vec{\xi}}{(2 \pi)^{3 / 2}} \mathcal{A}_{p}(\vec{\xi}) e^{-i \vec{\xi} \cdot \vec{w}}
$$

where normalization is such that $\mathcal{A}_{p}(\vec{\xi}=0)=1$; and $\Delta$ is the phase-matching function, which accounts for the conservation of longitudinal momentum in the microscopic PDC process,

$$
\Delta\left(\vec{w}_{1}, \vec{w}_{2}\right)=\left[k_{s z}\left(\vec{w}_{1}\right)+k_{s z}\left(\vec{w}_{2}\right)-k_{p z}\left(\vec{w}_{1}+\vec{w}_{2}\right)\right] l_{c},
$$

where $k_{s z}$ is the longitudinal component of the (ordinary) signal wave vector, $k_{p z}$ is the analogous quantity for the (extraordinary) pump, and $l_{c}$ is the crystal length.

We remark that the right-hand side of Eq. (6) is the firstorder term in the parameter $g$ of a perturbative expansion of the full solution of the propagation equation of field operators in the nonlinear crystal, so that expression (6) is valid only in the very low gain regime $g \ll 1$. Similarly, as it is well known, the right-hand side (r.h.s.) of Eq. (1) shows the zerothand first-order terms in $g$ of a perturbative expansion of the full PDC state.

By using the equivalence between the two formalisms, and by calculating the field correlation on the generic output state (1), we also obtain

$$
\begin{aligned}
\psi\left(\vec{w}_{1}, \vec{w}_{2}\right) & =\left\langle\psi_{\mathrm{PDC}}\left|A\left(\vec{w}_{1}, 0\right) A\left(\vec{w}_{2}, 0\right)\right| \psi_{\mathrm{PDC}}\right\rangle \\
& =\mathcal{C}\left(\vec{w}_{1}, \vec{w}_{2}\right)+\mathcal{C}\left(\vec{w}_{2}, \vec{w}_{1}\right)=2 \mathcal{C}\left(\vec{w}_{1}, \vec{w}_{2}\right)
\end{aligned}
$$

where we used the symmetry properties of the state. Thus, the two-photon state has the well-known form

$\left|\psi_{\mathrm{PDC}}\right\rangle=|0\rangle+\frac{1}{2} \int d \vec{w}_{1} \int d \vec{w}_{2} \psi\left(\vec{w}_{1}, \vec{w}_{2}\right) A^{\dagger}\left(\vec{w}_{1}\right) A^{\dagger}\left(\vec{w}_{2}\right)|0\rangle$,

with the biphoton amplitude $\psi$ being given by Eq. (6).

Apart from the biphoton amplitude, the other quantity of interest is the coherence function of the signal field, which after long but simple calculations can be derived from Eq. (10) as

$$
\begin{aligned}
G\left(\vec{w}, \vec{w}^{\prime}\right): & =\left\langle\psi_{\mathrm{PDC}}\left|A^{\dagger}(\vec{w}) A\left(\vec{w}^{\prime}\right)\right| \psi_{\mathrm{PDC}}\right\rangle \\
& =\int d \vec{w}_{2} \psi^{*}\left(\vec{w}, \vec{w}_{2}\right) \psi\left(\vec{w}^{\prime}, \vec{w}_{2}\right)
\end{aligned}
$$


From this equation, the total number of PDC photons is obtained as

$$
\begin{aligned}
N & =\int d \vec{w}_{1}\left\langle\psi_{\mathrm{PDC}}\left|A^{\dagger}\left(\vec{w}_{1}\right) A\left(\vec{w}_{1}\right)\right| \psi_{\mathrm{PDC}}\right\rangle \\
& =\int d \vec{w}_{1} \int d \vec{w}_{2}\left|\psi\left(\vec{w}_{1}, \vec{w}_{2}\right)\right|^{2} .
\end{aligned}
$$

In the ultralow gain regime where at most a single photon pair at a time is detected, one can address the question of how much the two photons of the pair are entangled. Contrary to typeII PDC, where twin photons are generated with orthogonal polarizations, in type I there is not such an obvious distinction between a signal and an idler field. A general and formally clean way of introducing a bipartition, which does not rely on any modal selection, is considering the two output modes of a symmetric beam splitter,

$$
\begin{aligned}
& A_{1}(\vec{w})=\frac{1}{\sqrt{2}}\left[A(\vec{w})+i a_{v}(\vec{w})\right], \\
& A_{2}(\vec{w})=\frac{1}{\sqrt{2}}\left[i A(\vec{w})+a_{v}(\vec{w})\right],
\end{aligned}
$$

where $a_{v}$ is a vacuum field operator. By substituting in the state (10) the inverse of Eq. (14), one gets two terms that describe the creation of two photons into each of the output modes 1 and 2 of the beam splitter, and a term that creates one photon in mode 1 and one photon in mode 2. Following the literature treating the degree of entanglement in PDC $[4,5,9,10]$, we shall consider, rather than the full PDC, the state vector conditioned to the measurement of a photon pair (the vacuum and two-photon terms are dropped). Assuming that detectors are placed at the two output modes, and coincidences are detected, the state conditioned to the appearance of a coincidence takes the form (apart from global normalization factors)

$$
|\phi\rangle=\int d \vec{w}_{1} \int d \vec{w}_{2} \psi\left(\vec{w}_{1}, \vec{w}_{2}\right) A_{1}^{\dagger}\left(\vec{w}_{1}\right) A_{2}^{\dagger}\left(\vec{w}_{2}\right)|0\rangle_{1}|0\rangle_{2} .
$$

The degree of entanglement of such a conditional state has been investigated in previous literature in the purely temporal $[4,5]$ or purely spatial [9-11] domains.

Notice that in practical implementations, twin photons could be sorted in various ways: for example, by their propagation directions (positive or negative with respect to any transverse axis) [23] or by their frequencies (smaller or larger than the central frequency). These methods are based on a modal selection and in practice would be efficient only for broad pump waists or quasimonocromatic pumps (because in these cases twin photons are created with symmetric transverse wave vectors $\pm \vec{q}$ and frequency offsets $\pm \Omega$, respectively). However, provided that one considers the state conditioned to the detection of a pair and that the pump is a quasi-plane-wave one, we expect, for these examples, results similar to those derived for the beam-splitter case.

\section{THE SCHMIDT NUMBER OF PDC ENTANGLEMENT: INTEGRAL FORMULA}

A good quantifier of the degree of entanglement for continuous variable pure states is the so-called Schmidt number, defined as the inverse of the purity of the state of each separate subsystem,

$$
\mathcal{K}=\frac{1}{\operatorname{Tr}\left\{\rho_{1}^{2}\right\}},
$$

where $\rho_{1}$ is the reduced density matrix of the subsystem 1 . In connection with the Schmidt decomposition of the PDC conditional state, the Schmidt number is recognized to give an estimate of the number of Schmidt modes participating in the entanglement, i.e., of the effective dimensionality of the entanglement [10].

We will derive an integral formula for the Schmidt number in the case of the conditional state (15), similar to what was obtained in Refs. [5,11]. First of all, the state (15) is not normalized,

$$
\langle\phi \mid \phi\rangle=\int d \vec{w}_{1} \int d \vec{w}_{2}\left|\psi\left(\vec{w}_{1}, \vec{w}_{2}\right)\right|^{2}=N .
$$

From the system conditional density matrix

$$
\rho=\frac{|\phi\rangle\langle\phi|}{\langle\phi \mid \phi\rangle},
$$

the reduced density matrix of the subsystem 1 can be calculated (Appendix A) as

$$
\begin{aligned}
\rho_{1} & =\operatorname{Tr}_{2}\{\rho\} \\
& =\frac{1}{N} \int d \vec{w}_{1} \int d \vec{w}_{1}^{\prime} G\left(\vec{w}_{1}^{\prime}, \vec{w}_{1}\right) A_{1}^{\dagger}\left(\vec{w}_{1}\right)|0\rangle_{1}{ }_{1}\langle 0| A_{1}\left(\vec{w}_{1}^{\prime}\right) .
\end{aligned}
$$

Notice that in the limit where the coherence function becomes a Dirac $\delta$ function, i.e., in the limit of a monochromatic planewave pump, the reduced density matrix becomes a sum of projectors onto one-photon states.

Next, we calculate the purity of such a reduced state:

$$
\operatorname{Tr}_{1}\left\{\rho_{1}^{2}\right\}=\frac{1}{N^{2}}\left[\int d \vec{w}_{1} \int d \vec{w}_{1}^{\prime}\left|G\left(\vec{w}_{1}, \vec{w}_{1}^{\prime}\right)\right|^{2}\right] .
$$

An integral formula for the Schmidt number can therefore be written as

$$
\mathcal{K}=\frac{N^{2}}{B},
$$

where

$$
\begin{aligned}
B= & \int d \vec{w}_{1} \int d \vec{w}_{1}^{\prime}\left|G\left(\vec{w}_{1}, \vec{w}_{1}^{\prime}\right)\right|^{2} \\
= & \int d \vec{w}_{1} \int d \vec{w}_{2} \int d \vec{w}_{1}^{\prime} \int d \vec{w}_{2}^{\prime}\left[\psi\left(\vec{w}_{1}, \vec{w}_{2}\right) \psi\left(\vec{w}_{1}^{\prime}, \vec{w}_{2}^{\prime}\right)\right. \\
& \left.\times \psi^{*}\left(\vec{w}_{1}, \vec{w}_{2}^{\prime}\right) \psi^{*}\left(\vec{w}_{1}^{\prime}, \vec{w}_{2}\right)\right]
\end{aligned}
$$

and $N$ is given by Eq. (17).

\section{THE NEARLY-PLANE-WAVE PUMP APPROXIMATION}

In order to evaluate the Schmidt number of the two-photon state from formula (21), we face the problem of calculating the six-dimensional and 12-dimensional integrals involved in the calculation of $N$ and $B$, respectively. These integrations can be numerically performed, but in the following we will provide more transparent results based on the approximation of a broad-enough pump profile. 
Let us come back to the expression (6) for the biphoton amplitude, which we rewrite as

$$
\begin{aligned}
\psi^{\prime}\left(\vec{w}_{1}, \vec{w}_{2}\right) & =\tilde{\mathcal{A}}_{p}\left(\vec{w}_{1}+\vec{w}_{2}\right) V\left(\vec{w}_{1}, \vec{w}_{2}\right), \\
V\left(\vec{w}_{1}, \vec{w}_{2}\right) & =\operatorname{sinc} \frac{\Delta\left(\vec{w}_{1}, \vec{w}_{2}\right)}{2} e^{i \frac{\Delta\left(\vec{w}_{1}, \vec{w}_{2}\right)}{2}},
\end{aligned}
$$

where $\Delta$ is the phase-matching function defined in Eq. (8), and we got rid of the constant $g /(2 \pi)^{3 / 2}$ that factors out in the ratio $\mathcal{K}=N^{2} / B$. We now introduce the pump spectral coordinates $\vec{w}_{p}=\vec{w}_{1}+\vec{w}_{2}:=\left(\vec{q}_{p}, \Omega_{p}\right)$. Provided that $\sigma_{p}$ is the transverse waist of the pump beam at the output crystal face, and $\tau_{p}$ is its duration, the pump Fourier transform $\tilde{\mathcal{A}}_{p}$ dies out on the scales $\delta q_{p}=2 / \sigma_{p}, \delta \Omega_{p}=2 / \tau_{p}$. This claim is exactly true for a Gaussian pump profile,

$$
\begin{gathered}
\mathcal{A}_{p}(\vec{x}, t)=e^{-x^{2} / \sigma_{p}^{2}} e^{-t^{2} / \tau_{p}^{2}}, \\
\tilde{\mathcal{A}}_{p}\left(\vec{q}_{p}, \Omega_{p}\right)=\frac{\sigma_{p}^{2} \tau_{p}}{2^{3 / 2}} e^{-q_{p}^{2} \sigma^{2} / 4} e^{-\Omega_{p}^{2} \tau_{p}^{2} / 4} .
\end{gathered}
$$

The function $V$ is strongly peaked along the curve where phase matching takes place. As elaborated in detail in the Appendix B of Ref. [20], for a broad-enough pump, the variation of this function with respect to the pump spectral coordinates can be neglected. In other words, $V\left(\vec{w}_{1},-\vec{w}_{1}+\vec{w}_{p}\right)$ does not vary significantly with $\vec{w}_{p}$ on the scale over which the pump Fourier profile dies out:

$$
\begin{aligned}
\tilde{\mathcal{A}}_{p}\left(\vec{w}_{p}\right) V\left(\vec{w}_{1},-\vec{w}_{1}+\vec{w}_{p}\right) & \approx \tilde{\mathcal{A}}_{p}\left(\vec{w}_{p}\right) V\left(\vec{w}_{1},-\vec{w}_{1}\right) \\
& :=\tilde{\mathcal{A}}_{p}\left(\vec{w}_{p}\right) V\left(\vec{w}_{1}\right) .
\end{aligned}
$$

We call this approximation the nearly-plane-wave pump approximation (NPWPA). It is based on making a Taylor expansion of $V$ in a power series of the pump variables $\vec{w}_{p}$, and on finding the conditions under which the first-order terms of the expansion can be neglected with respect to the zeroth-order term [20]. These conditions can be summarized as

$$
\begin{gathered}
\tau_{p} \gg \tau_{\mathrm{GVM}}=\left|\frac{l_{c}}{v_{g s}}-\frac{l_{c}}{v_{g p}}\right|, \\
\sigma_{p} \gg l_{\text {walk-off }}=\left|l_{c} \frac{\partial k_{p}}{\partial q_{x}}\right| .
\end{gathered}
$$

Here, $\tau_{\mathrm{GVM}}$ is the maximum delay time between the signal and the pump wave in crossing the nonlinear crystal due to the mismatch between the group velocities $v_{g s}, v_{g p}$ of the ordinary signal and extraordinary pump. $l_{\text {walk-off }}$ is the maximum lateral walk-off between the two waves, associated with the tilt of the Poynting vectors. In the example of a $4 \mathrm{~mm}$ beta barium borate (BBO) crystal, pumped at a wavelength $\lambda_{p}=527 \mathrm{~nm}$, we have $\tau_{\mathrm{GVM}} \approx 500 \mathrm{fs}, l_{\text {walk-off }} \approx 250 \mu \mathrm{m}$, so that the NPWPA is within reach of the practical experimental generation of PDC photon pairs.

The use of this limit remarkably simplifies the expression (21) of the Schmidt number. As reported in detail in Appendix $\mathrm{B}$, the integral formula (21) takes the form

$$
\mathcal{K}=\frac{N^{2}}{B} \rightarrow \frac{\left[\int d \vec{\xi}_{p}\left|\mathcal{A}_{p}\left(\vec{\xi}_{p}\right)\right|^{2}\right]^{2}}{\int d \vec{\xi}_{p}\left|\mathcal{A}_{p}\left(\vec{\xi}_{p}\right)\right|^{4}} \frac{\left[\int d \vec{w}|V(\vec{w})|^{2}\right]^{2}}{(2 \pi)^{3} \int d \vec{w}|V(\vec{w})|^{4}}
$$

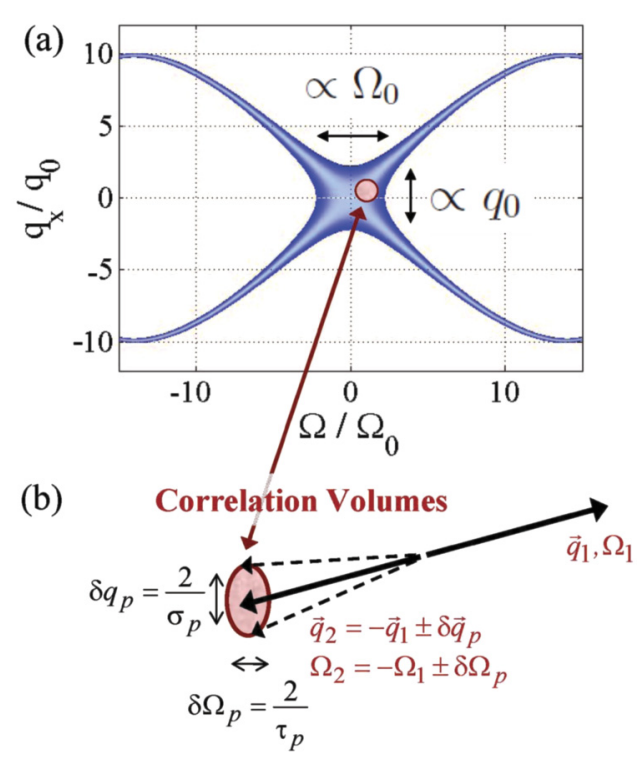

FIG. 1. (Color online) Geometrical interpretation of the Schmidt number. (a) The phase-matching region in the $\left(q_{x}, \Omega\right)$ plane, here defined by $|V(\vec{q}, \Omega)|^{2}>0.1$ (the full $3 \mathrm{D}$ volume has a biconical shape). (b) The correlation volumes, which in the 3D picture would be Gaussian bullets of size determined by the spectral extension $\delta q_{p}^{2} \delta \Omega_{p}$ of the pump. The case of a $4 \mathrm{~mm}$ type-I BBO crystal, pumped at $\lambda_{0}=527 \mathrm{~nm}$ for collinear phase matching.

The integrals involving the pump coordinates can now be readily performed. By assuming a Gaussian pump profile as in Eq. (26), we easily obtain

$$
\mathcal{K}=\frac{\int d \vec{w}|V(\vec{w})|^{2}}{\int d \vec{w}|V(\vec{w})|^{4}} \frac{\int d \vec{w} \operatorname{sinc}^{2} \frac{\Delta(\vec{w})}{2}}{\pi^{\frac{3}{2}} \frac{4}{\sigma_{p}^{2}} \frac{2}{\tau_{p}}} .
$$

As we shall see in the following, under rather general conditions, ${ }^{2}$ the first term on the r.h.s. of Eq. (32) is a purely numerical factor, namely,

$$
\frac{\int d \vec{w}|V(\vec{w})|^{2}}{\int d \vec{w}|V(\vec{w})|^{4}} \approx \frac{3}{2} .
$$

Thanks to this circumstance, the result of Eq. (32) has a transparent geometrical interpretation. On the one hand, the term $\int d \vec{w} \operatorname{sinc}^{2} \frac{\Delta(\vec{w})}{2}$ can be interpreted as the volume of the region in the $(\vec{q}, \Omega)$ 3D space where phase matching occurs, since the $\operatorname{sinc}^{2}$ function has a sharp maximum where $\Delta(\vec{q}, \Omega)=0$ (see Fig. 1). This corresponds to the portion of the $(\vec{q}, \Omega)$ space where the probability of photon-pair production is not negligible. On the other hand, the quantity $\frac{4}{\sigma_{p}^{2}} \frac{2}{\tau_{p}}$ at the denominator of Eq. (32) represents the spectral volume of the pump $\delta q_{p}^{2} \delta \Omega_{p}=\frac{4}{\sigma_{p}^{2}} \frac{2}{\tau_{p}}$. This quantity defines the correlation volume, i.e., the size the of the correlated modes, because the expression (24) tells us that in the NPWPA, the width of the biphoton correlation as a function of $\vec{w}_{1}+\vec{w}_{2}$ is determined by the pump Fourier profile. Thus, $\delta q_{p}^{2} \delta \Omega_{p}$ represents the

\footnotetext{
${ }^{2}$ This condition amounts to requiring that phase matching occurs within the spectral region considered, with a counterexample being provided in Sec. VII.
} 
uncertainty in the determination of the transverse wave vector $\vec{q}_{2}$ and frequency $\Omega_{2}$ of a photon, once the transverse wave vector $\vec{q}_{1}$ and frequency $\Omega_{1}$ of its twin have been determined. Therefore, $\mathcal{K}$ is on the order of the ratio

$$
\mathcal{K} \propto \frac{\text { volume of the phase-matching region }}{\text { correlation volume }},
$$

and can be interpreted as the number of correlated modes participating to the state. The formula (34) gives us a simple geometric interpretation of the Schmidt number, which will be very useful in understanding some results.

\section{SCHMIDT NUMBER OF 3D $X$-ENTANGLED BIPHOTONS}

We can proceed further and find an analytical approximation of the Schmidt-number result of Eq. (32) in the NPWPA. To this end, we need to calculate integrals over the phasematching curves of the form $\int d \vec{w}|V(\vec{w})|^{2}, \int d \vec{w}|V(\vec{w})|^{4}$. Our calculations are based on the use of two further approximations (in additions to the NPWPA):

(i) The first approximation consists of a quadratic expansion of the phase-mismatch function with respect to $q$ and $\Omega$, which is equivalent to adopting the paraxial and quadratic dispersion approximations:

$$
\Delta(q, \Omega)=\Delta_{0} l_{c}-\frac{q^{2}}{q_{0}^{2}}+\frac{\Omega^{2}}{\Omega_{0}^{2}},
$$

where $\Delta_{0}=2 k_{s}-k_{p}$ is the collinear phase mismatch at degeneracy, and

$$
\begin{gathered}
q_{0}=\sqrt{\frac{k_{s}}{l_{c}}}, \\
\Omega_{0}=\sqrt{\frac{1}{k_{s}^{\prime \prime} l_{c}}},
\end{gathered}
$$

with $k_{s}=k_{s}(0), k_{s}^{\prime \prime}=d^{2} k_{s} /\left.d \Omega^{2}\right|_{0}$. This expansion is strictly valid only for small $\Omega$ (close to degeneracy) and small $q$. For the remainder of this section, we assume conditions of collinear phase matching, $\Delta_{0} l_{c} \approx 0$, where the phase-matching curve in the plane $(q, \Omega)$ has the characteristic hyperbolic geometry shown in Fig. 1 . The parameters $q_{0}$, associated with spatial diffraction, and $\Omega_{0}$, associated with the group velocity dispersion (GVD), define the typical variation scale of $|V(\vec{w})|^{2}$ along $q$ and $\Omega$, respectively. In the example of the $4 \mathrm{~mm} \mathrm{BBO}$ crystal, their values are $q_{0} \approx 5 \times 10^{-2} \mu \mathrm{m}^{-1}$, $\Omega_{0} \approx 0.76 \times 10^{14} \mathrm{~Hz}$.

(ii) The second approximation consists of substituting the $\operatorname{sinc}^{2}\left[\frac{\Delta(\vec{w})}{2}\right]$ with a box function, with the same value of the indefinite integral,

$$
\operatorname{sinc}^{2}\left[\frac{\Delta(\vec{w})}{2}\right] \rightarrow \chi_{\alpha}\left[\frac{\Delta(\vec{w})}{2}\right]=\left\{\begin{array}{l}
\frac{\pi}{\alpha}, \frac{\Delta(\vec{w})}{2} \in\left(-\frac{\alpha}{2} ; \frac{\alpha}{2}\right) \\
0, \text { elsewhere, }
\end{array}\right.
$$

which satisfies $\int \operatorname{sinc}^{2}(x) d x=\int \chi_{\alpha}(x) d x=\pi$. Here the parameter $\alpha$ can be used, in principle, as a fitting parameter. Approximation (38) seems very rough, but it turned out surprisingly accurate: the rationale behind this result is that the $\operatorname{sinc}^{2}$ has a sharp peak where $\Delta(\vec{w})=0$, and in order to evaluate its integral in the $3 \mathrm{D}$ space, it is more important to
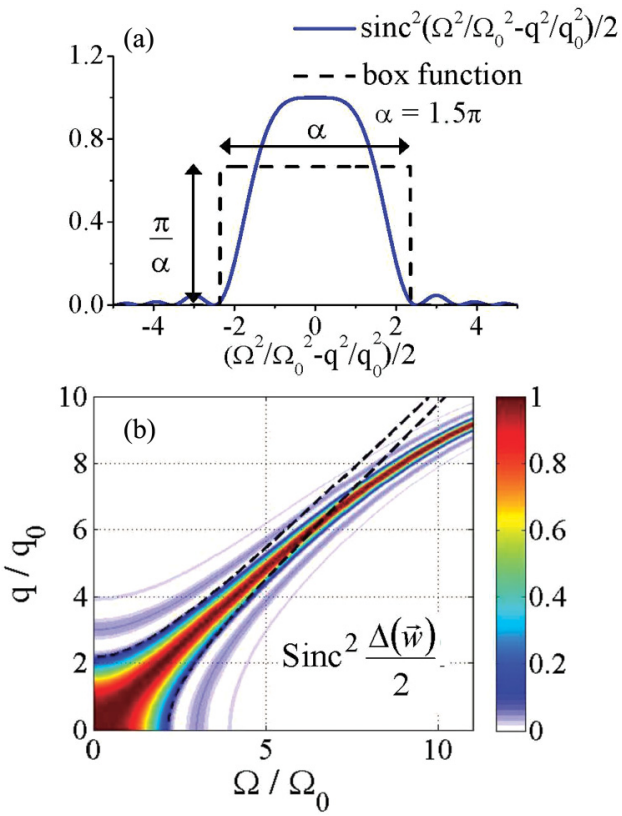

FIG. 2. (Color online) (a) Box function approximation (38) of the $\operatorname{sinc}^{2}$ function. (b) Comparison between the true phase-matching function $|V(\vec{q}, \Omega)|^{2}$ and its box function approximation (boundaries of the box function are shown by dashed lines). Collinear phasematching case $\left(\Delta_{0} l_{c}=0, \theta_{p}=22.934^{\circ}\right), l_{c}=4 \mathrm{~mm}$.

take into account the geometrical shape of the curve where its maximum lies rather than the detailed shape of the peak. Figure 2 compares the box function approximation to the true phase-matching function in the example of the BBO crystal. Here, substantial deviations appear at large values of $\Omega$ and $q$ because of the failure of the quadratic approximation for phase matching.

Notice that if we also assume that $|V(\vec{w})|^{4}$ can be approximated by the box function $\chi_{\alpha}^{2}(\vec{w})$, with the request that, this time,

$$
\int d x \chi_{\alpha}^{2}(x)=\frac{\pi^{2}}{\alpha}=\int d x \operatorname{sinc}^{4}(x)=\frac{2}{3} \pi,
$$

then we find the correct value of $\alpha=\frac{3}{2} \pi$. This also shows that within these approximations, the ratio

$$
\frac{\int d \vec{w}|V(\vec{w})|^{2}}{\int d \vec{w}|V(\vec{w})|^{4}} \approx \frac{\int d \vec{w} \chi_{\alpha}(\vec{w})}{\int d \vec{w} \chi_{\alpha}^{2}(\vec{w})}=\frac{\alpha}{\pi}=\frac{3}{2} .
$$

The box function approximation allow us to evaluate easily the integrals over the phase-matching curves inside Eq. (32). In this evaluation, we assume that our model describes a measurement performed over a large but limited spectral bandwidth $\Omega \in\left(-\Omega_{\max }, \Omega_{\max }\right)$. For simplicity, here we do not pose limits to the spatial bandwidth (which will be instead done in the following section). After some calculations, we obtain the following two different results depending on the detected bandwidth, $\bar{\Omega}_{\max }=\frac{\Omega_{\max }}{\Omega_{0}}$ :

(i) small bandwidth result $\left(\bar{\Omega}_{\max }<\sqrt{\alpha}\right)$,

$$
\mathcal{K}=\frac{\alpha}{4} \sqrt{\frac{\alpha}{\pi}} q_{0}^{2} \Omega_{0} \sigma_{p}^{2} \tau_{p}\left[\frac{\bar{\Omega}_{\max }}{\sqrt{\alpha}}+\frac{1}{3}\left(\frac{\bar{\Omega}_{\max }}{\sqrt{\alpha}}\right)^{3}\right] ;
$$


(ii) large bandwidth result $\left(\bar{\Omega}_{\max }>\sqrt{\alpha}\right)$,

$$
\mathcal{K}=\frac{\alpha}{2} \sqrt{\frac{\alpha}{\pi}} q_{0}^{2} \Omega_{0} \sigma_{p}^{2} \tau_{p}\left(\frac{\bar{\Omega}_{\max }}{\sqrt{\alpha}}-\frac{1}{3}\right) .
$$

First of all, we observe that the condition on the bandwidth can be roughly reformulated as $\Omega_{\max }$ being smaller or bigger than the characteristic GVD bandwidth $\Omega_{0}$ ( since $\alpha$ is on the order of unity). The small-bandwidth case corresponds to the situation where the portion of PDC emission intercepted by the measurement lies within the central region of the phasematching curve (see Fig. 1), where the phase matching has no hyperbolic structure. Equations (41) and (42) tell us that in both cases, the Schmidt number is proportional to the number of modes contained in a unit volume of the phase-matching region:

$$
\mathcal{K} \propto \frac{\pi q_{0}^{2} \Omega_{0} \sigma_{p}^{2} \tau_{p}}{8}=\frac{\pi q_{0}^{2} \Omega_{0}}{\delta \vec{q}_{p}^{2} \delta \Omega_{p}} .
$$

However, as the detected bandwidth increases beyond the GVD bandwidth $\Omega_{0}$, the hyperbolic geometry of phase matching enters into play, and the Schmidt-number result of Eq. (42) shows a linear increase with the bandwidth.

We remind the reader that the analytical expressions (41) and (42) estimate the Schmidt number within the NPWPA and the quadratic approximation, expressed by the condition (28) and (35), respectively. In order to verify its validity, and at the same time provide a numerical estimation of $\mathcal{K}$ in regions of the parameter space where the NPWPA does not hold, we performed a numerical evaluation of the general expression of $\mathcal{K}$ given by Eqs. (13), (21), and (24). As it involves a six-dimensional integral for the evaluation of $N$ and a 12-dimensional integral for the evaluation of $B$, the use of a Monte Carlo integration is mandatory. We used the well-known method of importance sampling [28] with the aim of improving the efficiency of the Monte Carlo algorithm by increasing the density of the sampled points where the functions under the integrals are larger. A natural choice has been to sample some of the Fourier variables [namely, the "pump" variables in Eq. (B5)] according to Gaussian distributions coincident with the Gaussian pump spectral amplitude (27), which in the NPWPA represents the narrowest factor of the biphoton amplitude in Eq. (24). The implemented algorithm, which is very efficient for narrow spectral pump profiles, allows the evaluation of $B$ and $N$ even in the region where the NPWP approximation fails. No other substantial approximations are introduced, as the phase-matching function is here evaluated by means of the empirical Sellmeier formulas [29].

Figure 3 compares the analytic result of Eqs. (41) and (42) with the Monte Carlo numerics, performed both without approximation (squares) and with the quadratic approximation for phase matching (triangles). Figure 3(a) is plotted for parameters of the pump within the NPWP approximation (although very reasonable for an experimental realization) and shows excellent agreement between the analytical curve and the numerics, in the range of validity of the quadratic approximation. Indeed, the analytic result follows a Monte Carlo simulation performed with the quadratic approximation very well, showing that the box function approximation captures the basic geometrical properties of the phase-matching
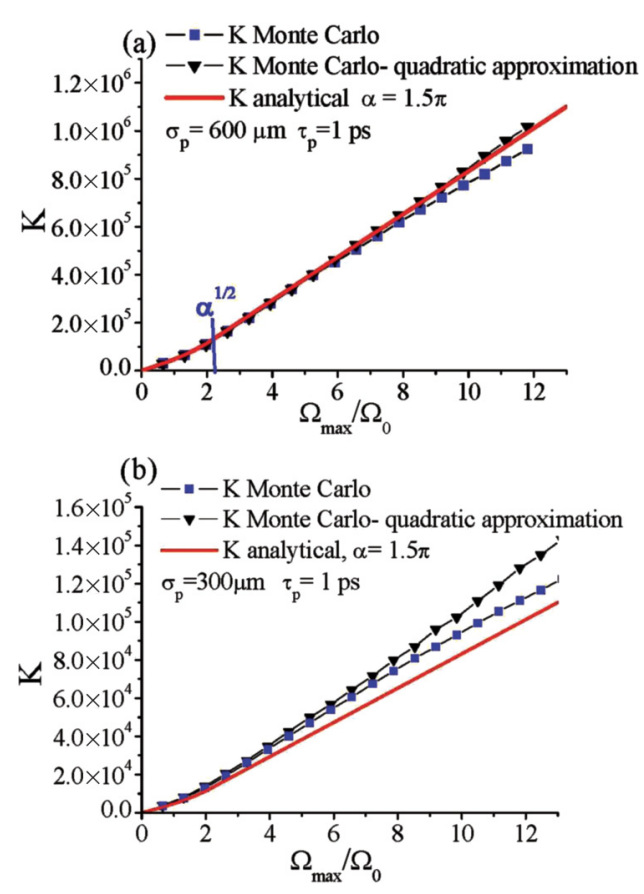

FIG. 3. (Color online) Schmidt-number results. Comparison between the analytic formulas (41) and (42) (solid red line) and the Monte Carlo simulations, without any approximation (squares) and with quadratic approximation (triangles). (a) Pump parameters are within the NPWPA. (b) Focused pump, beyond the NPWPA. Collinear phase matching $\left(\Delta_{0} l_{c}=0, \theta_{p}=22.934^{\circ}\right), l_{c}=4 \mathrm{~mm}$.

function. In Fig. 3(b), the pump beam is more focused and, as expected, the analytic result deviates from numerics because of the failure of the NPWP approximation.

Monte Carlo calculations permit one to obtain results also in the region of parameters beyond the NPWP approximation. Figure 4 plots the Schmidt number as a function of the parameter $\beta=\delta q_{p}^{2} \delta \Omega_{p} / q_{0}^{2} \Omega_{0}$. The NPWP approximation is valid only for $\beta \ll 1$, i.e., when the widths $\delta \Omega_{p}$, $\delta q_{p}$ of the pump Fourier profile are much smaller than the characteristic

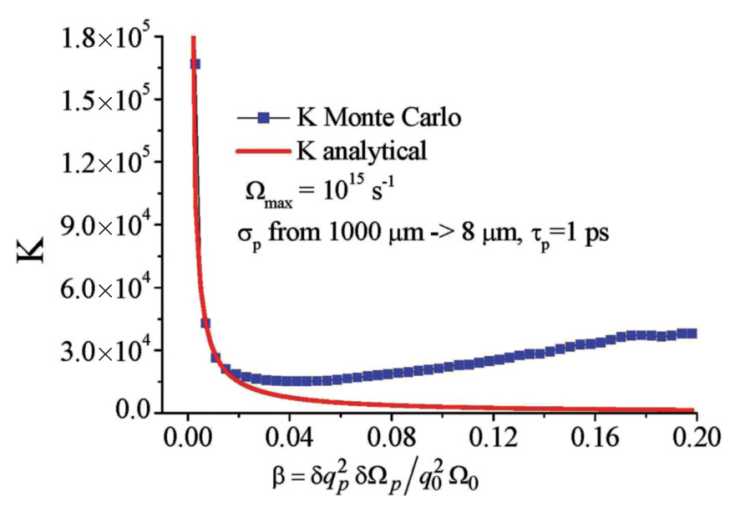

FIG. 4. (Color online) Schmidt number as a function of $\beta=$ $\delta q_{p}^{2} \delta \Omega_{p} / q_{0}^{2} \Omega_{0}$. The blue squares plot the result of a Monte Carlo calculation, without any approximation, and show that $\mathcal{K}$ after reaching a minimum increases again almost linearly with $\beta$. The red solid line is the analytic result, decreasing as $1 / \beta$ [see Eq. (42)], valid only within the NPWPA (small $\beta$ ). Collinear phase matching $\left(\Delta_{0} l_{c}=0, \theta_{p}=22.934^{\circ}\right), l_{c}=4 \mathrm{~mm}$. 
scales of variation of phase matching $\Omega_{0}, q_{0}$, respectively. ${ }^{3}$ The Monte Carlo result shows a decrease of the Schmidt number as $1 / \beta$ for $\beta \ll 1$, as predicted by the analytic result (42) in the NPWPA (red solid line in the figure). However, after reaching a minimum, the Schmidt number increases again almost linearly with $\beta$. This behavior is very similar to that predicted in a purely spatial model of PDC in Ref. [9] and can be understood as follows: for a broad pump, when the NPWPA is valid, the width of the correlation is determined by the pump Fourier profile, and the number of spatiotemporal modes can be estimated as in formula (34) as being proportional to the volume of phase matching divided by the pump spectral volume, $\mathcal{K} \propto q_{0}^{2} \Omega_{0} / \delta q_{p}^{2} \delta \Omega_{p}=\frac{1}{\beta}$. For a very focused pump, instead, the phase-matching function in the $\vec{q}$ direction has a smaller scale of variation than the pump Fourier spatial profile, so that the width of correlation is rather determined by the characteristic width $\vec{q}_{0}$ of phase matching, and we can suppose that the number of modes is now $\mathcal{K} \propto \delta q_{p}^{2} / q_{0}^{2} \propto \beta$.

\section{FACTORABILITY OF THE SCHMIDT NUMBER IN ITS TEMPORAL AND SPATIAL COMPONENTS}

In the literature, the Schmidt dimensionality of twin photons is often calculated within models of PDC restricted to the spatial or the temporal domain (see Refs. $[4,5,9,10]$ ). The nonfactorable character of the spatiotemporal correlation demonstrated in Refs. [19,20] suggests to us that the full 3D spatiotemporal Schmidt number is not trivially given by the product of the spatial 2D and the temporal 1D Schmidt numbers. In this section, we would like to understand this point.

To this end, we consider models for PDC in lower dimensionalities, and follow the same procedure outlined in the previous sections to calculate the Schmidt number. The purely spatial 2D model is obtained by neglecting the temporal coordinate and setting $\Omega=0$. Similarly, the purely temporal $1 \mathrm{D}$ model neglects the spatial coordinates and sets $\vec{q}=0$. The starting point of the analysis is, in both cases, the general integral formula for the Schmidt number (21), where we have now to interpret the Fourier coordinates as

$$
\vec{w}=\left\{\begin{array}{lll}
\Omega \in \mathcal{R} & \text { in } & \text { 1D } \\
\vec{q} \in \mathcal{R}^{2} & \text { in } & \text { 2D } \\
\vec{q}, \Omega \in \mathcal{R}^{3} & \text { in } & \text { 3D. }
\end{array}\right.
$$

Similarly, in the expression involving the coordinates in the direct space,

$$
\vec{\xi}=\left\{\begin{array}{lll}
t \in \mathcal{R} & \text { in } & 1 \mathrm{D} \\
\vec{x} \in \mathcal{R}^{2} & \text { in } & 2 \mathrm{D} \\
\vec{x}, t \in \mathcal{R}^{3} & \text { in } & 3 \mathrm{D} .
\end{array}\right.
$$

For example, by introducing the NPWP approximation in the various models (clearly NPWPA in the spatial model

\footnotetext{
${ }^{3}$ Actually, the limits of validity of the NPWPA expressed by (29) and (30) are typically much more restrictive than $\beta \ll 1$. For example, for the BBO crystal considered here, $\delta q_{p}<q_{0}$ implies roughly $\sigma_{p}>2 l_{\text {diff }}=2 / q_{0} \approx 40 \mu \mathrm{m}$, while $\delta \Omega_{p}<\Omega_{0}$ implies $\tau_{p}>$ $2 \tau_{\mathrm{GVD}}=2 / \Omega_{0} \approx 26 \mathrm{fs}$.
}

means that the pump has a broad waist, while the temporal model assumes a long-enough pulse duration), we obtain the NPWPA expression for the Schmidt number in an arbitrary $D$-dimensional model:

$$
\mathcal{K}=\frac{\left[\int d \vec{\xi}_{p}\left|\mathcal{A}_{p}\left(\vec{\xi}_{p}\right)\right|^{2}\right]^{2}}{\int d \vec{\xi}_{p}\left|\mathcal{A}_{p}\left(\vec{\xi}_{p}\right)\right|^{4}} \frac{\left[\int d \vec{w}|V(\vec{w})|^{2}\right]^{2}}{(2 \pi)^{D} \int d \vec{w}|V(\vec{w})|^{4}} .
$$

\section{A. Spatial Schmidt number $\mathcal{K}_{2 \mathrm{D}}$}

By performing calculations similar to those reported for the 3D model, we derive an expression for the Schmidt number in the purely spatial case, valid within the NPWPA and the quadratic approximation for phase matching. The latter one corresponds to approximating the phase-matching function as

$$
\Delta_{2 \mathrm{D}}(\vec{q})=k_{s}(\vec{q})+k_{s}(-\vec{q})-k_{p} \approx \frac{q^{2}}{q_{0}^{2}} .
$$

In the $2 \mathrm{D}$ case, the result depends on the spatial bandwidth $\bar{q}_{\max }=q_{\max } / q_{0}$ intercepted by the measurement

$$
\begin{gathered}
\mathcal{K}_{2 \mathrm{D}}=\frac{3}{8} \pi \sigma_{p}^{2} q_{0}^{2} \frac{\bar{q}_{\max }^{2}}{\alpha}, \quad \bar{q}_{\max }<\sqrt{\alpha} \\
\mathcal{K}_{2 \mathrm{D}}=\frac{3}{8} \pi \sigma_{p}^{2} q_{0}^{2}, \quad \bar{q}_{\max }>\sqrt{\alpha},
\end{gathered}
$$

where we remind the reader that $\alpha \approx 1.5 \pi$.

In Fig. 5, this curve is compared with an exact Monte Carlo calculation performed in the $2 \mathrm{D}$ model. Beyond noticing that the two results agree qualitatively, we remark that differently from the 3D case, the 2D Schmidt number saturates to the maximum value, $\mathcal{K}_{2 \mathrm{Dmax}}=\frac{3}{8} \pi \sigma_{p}^{2} q_{0}^{2}=\frac{3}{2} \pi q_{0}^{2} / \delta q_{p}^{2}$. This behavior can be explained with the help of the geometrical interpretation (34), valid in the NPWPA, which evaluates the Schmidt number as the ratio between the volume of the phase-matching region and the correlation volume. In the $2 \mathrm{D}$ case, phase matching is described by Eq. (47), so that in the $\left(q_{x}, q_{y}\right)$ plane, phase matching occurs within a circle of area $\approx \pi \vec{q}_{0}^{2}$. For increasing $q_{\max }$, the PDC photons are detected in increasingly large circular regions, so that the Schmidt number increases quadratically with $q_{\max }$ until the border of the phase matching region, $q_{\max }=q_{0} \sqrt{\alpha}$, is reached.

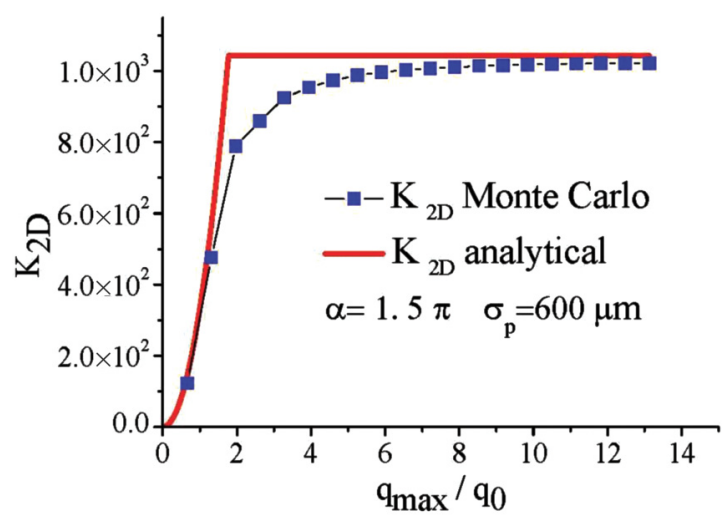

FIG. 5. (Color online) 2D spatial Schmidt number $\mathcal{K}_{2 \mathrm{D}}$ as a function of the collected spatial bandwidth $q_{\max }$. The solid red line is the analytic result of Eqs. (48) and (49); the squares plot the Monte Carlo numeric result. The waist of the pump beam is $\sigma_{p}=600 \mu \mathrm{m}$. 


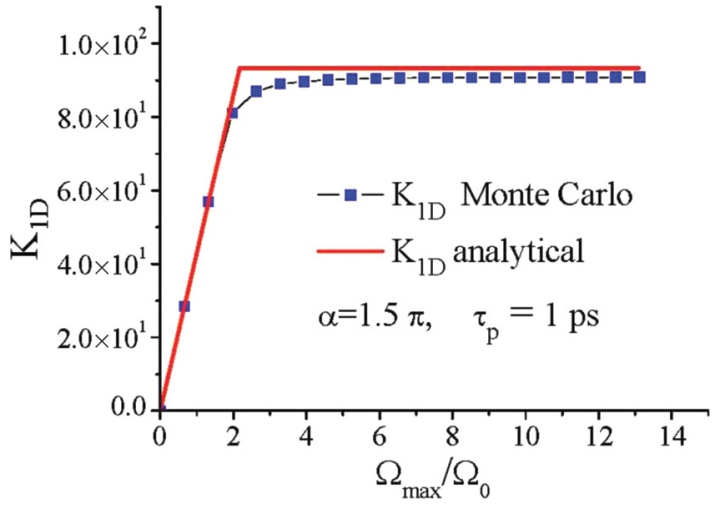

FIG. 6. (Color online) 1D temporal Schmidt number $\mathcal{K}_{1 \mathrm{D}}$ as a function of the maximum temporal frequency $\Omega_{\max }$. The solid line shows the analytic result (within the NPWPA and quadratic approximation); the squares plot the numeric exact result. Pump time duration $\tau_{p}=1 \mathrm{ps}$.

\section{B. Temporal Schmidt number $\mathcal{K}_{1 \mathrm{D}}$}

We now consider the purely temporal model of PDC by setting $\vec{q}=0$. We perform again the analytic calculation in the NPWPA and the quadratic approximation for phase matching, which in the $1 \mathrm{D}$ case reads

$$
\Delta_{1 \mathrm{D}}(\Omega)=k_{s}(\Omega)+k_{s}(-\Omega)-k_{p} \approx \frac{\Omega^{2}}{\Omega_{0}^{2}} .
$$

The analytic expression for $\mathcal{K}_{1 \mathrm{D}}$ in these limits, obtained by using the box function approximation, depends on the collected temporal bandwidth, $\bar{\Omega}_{\max }=\Omega_{\max } / \Omega_{0}$ :

$$
\begin{gathered}
\mathcal{K}_{1 \mathrm{D}}=\sqrt{\frac{\alpha}{\pi}} \tau_{p} \Omega_{0} \frac{\bar{\Omega}_{\max }}{\sqrt{\alpha}}, \bar{\Omega}_{\max }<\sqrt{\alpha} \\
\mathcal{K}_{1 \mathrm{D}}=\sqrt{\frac{\alpha}{\pi}} \tau_{p} \Omega_{0}, \bar{\Omega}_{\max }>\sqrt{\alpha} .
\end{gathered}
$$

Figure 6 plots this analytical result together with the exact Monte Carlo 1D calculation. Also in this case, similarly to the $2 \mathrm{D}$ case, the Schmidt number saturates to the maximum value, $\mathcal{K}_{1 \text { Dmax }} \approx \tau_{p} \Omega_{0}$, because phase matching occurs only inside an interval of size $\approx \Omega_{0}$, so that by increasing $\Omega_{\max }$ beyond the critical value $\sqrt{\alpha} \Omega_{0}$, we begin to consider regions where there is no phase matching, which do not contribute to the integral.

\section{Comparison}

We can now compare the results obtained in the models of various dimensionalities. To this end, we have to slightly reformulate the $3 \mathrm{D}$ problem. In Sec. V, we calculated $\mathcal{K}$ as a function of the collected temporal bandwidth by assuming that no selection on the spatial bandwidth was performed, i.e., $\bar{q}_{\max }=\infty$. This is a possible correct choice to present results, but in order to have a clean comparison with the 2D and 1D models, we also need to limit the detected spatial bandwidth. The simplest possibility is to set $\bar{q}_{\max }=\bar{\Omega}_{\max }$. This choice is justified by the fact that in the quadratic approximation, phase matching is realized along the lines $\frac{q}{q_{0}}= \pm \frac{\Omega}{\Omega_{0}}$ so that by increasing simultaneously the spatial and temporal bandwidth, $\frac{q_{\max }}{q_{0}}=\frac{\Omega_{\max }}{\Omega_{0}}$, one follows the phase-matching curve.

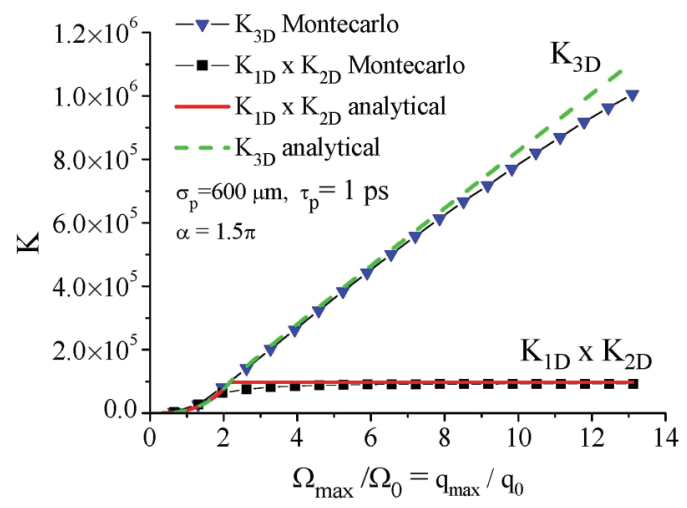

FIG. 7. (Color online) Comparison between the Schmidt number $\mathcal{K}$ calculated in the full spatiotemporal model and the product of the Schmidt numbers, $\mathcal{K}_{1 \mathrm{D}} \times \mathcal{K}_{2 \mathrm{D}}$, calculated in the purely $1 \mathrm{D}$ temporal and 2D spatial models. The abscissa is the collected temporal bandwidth, set equal to the spatial bandwidth in normalized units. Lines plot analytic results; symbols provide the Monte Carlo results.

With this in mind, analytical calculations in the NPWPA, the quadratic approximation for phase matching, and the box function approximation can be performed. The result for the 3D Schmidt number is plotted in Fig. 7 (dashed line) together with the Monte Carlo exact result (triangles). In the same figure, we compare these 3D results with the product of the Schmidt numbers obtained in the models with lower dimensionalities, i.e., $\mathcal{K}_{2 \mathrm{D}} \times \mathcal{K}_{1 \mathrm{D}}$. From these plots, it clearly emerges that the factorizability holds only when the detected bandwidth is small, i.e., when both $q_{\max }$ and $\Omega_{\max }$ lie within the phase-matching bandwidths $q_{0}, \Omega_{0}$, respectively. However, as the detected bandwidth gets larger, the result in the fully 3D spatiotemporal model grows linearly with the detected bandwidth and diverges clearly from the product $\mathcal{K}_{2 \mathrm{D}} \times \mathcal{K}_{1 \mathrm{D}}$, which saturates to a fixed value $\propto q_{0}^{2} \Omega_{0}$. This result can be easily understood with the help of the geometrical interpretation of the Schmidt number as the number of entangled modes contained in the phase-matching region: close to the degeneracy and to the collinear emission, the phase-matching region can be seen as a spherical region, which obviously factorizes in the spatial and temporal components, so that the number of spatiotemporal modes is trivially the product of the numbers of spatial and temporal mode times. Conversely, if the collected bandwidth is large enough, then the biconical, nonfactorizable geometry of phase matching comes into play, so that a full 3D model has to be used to correctly compute the number of spatiotemporal modes.

\section{ENTANGLEMENT WITHOUT PHASE MATCHING}

Until now, we considered the case of collinear phase matching $\left(\Delta_{0} l_{c}=0\right)$, characterized by the fact that exact phase matching $\Delta(\vec{w})=0$ can be realized for any value of the transverse wave vector of the photon pair, such that $q / q_{0}= \pm \Omega / \Omega_{0}$. However, if the crystal is tuned away from the collinear conditions $\left(\Delta_{0} l_{c} \neq 0\right)$, then there exist regions of the $(\vec{q}, \Omega)$ space where phase matching does not occur at all. In these regions, the probability of emission of photon pairs is low, although not zero. 


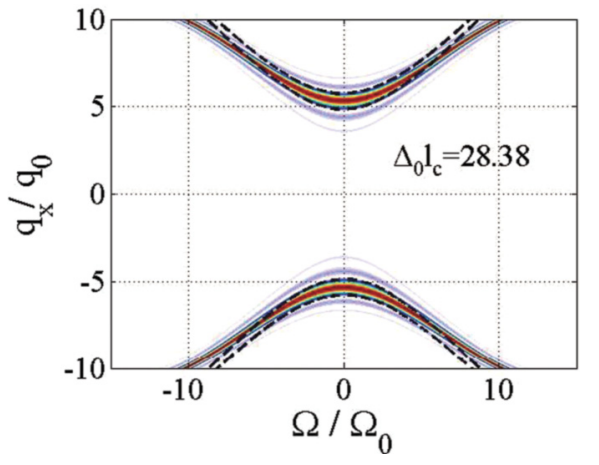

FIG. 8. (Color online) Color map of $|V(\vec{q}, \Omega)|^{2}$ in the noncollinear phase-matching case, $\Delta_{0} l_{c}>0\left(\Delta_{0} l_{c}=23.38\right)$. The dashed lines are the boundaries of the box function, where $\Delta(\vec{q}, \Omega)= \pm \alpha$.

In particular, we focus on the case of noncollinear phase matching $\left(\Delta_{0} l_{c}>0\right)$. Figure 8 shows the typical behavior of the phase-matching function $\operatorname{sinc}^{2}[\Delta(\vec{w}) / 2]$. From the quadratic expansion of the phase mismatch,

$$
\Delta(q, \Omega) \approx \Delta_{0} l_{c}-\frac{q^{2}}{q_{0}^{2}}+\frac{\Omega^{2}}{\Omega_{0}^{2}},
$$

we immediately realize that phase matching does not occur for modes close to collinear emission, namely, having $|\vec{q}|<$ $\sqrt{\Delta_{0} l_{c}}$

We have calculated the 3D spatiotemporal Schmidt number in these conditions: Fig. 9 plots the result as a function of the collected spatial bandwidth $q_{\max }$. We find that for small bandwidths, such that the collected photons are not phase matched, the degree of entanglement is very high, and is actually larger than in the region where phase matching is realized. This result is apparently paradoxical because, in the absence of phase matching, the probability of emission of a photon pair is very low and one could infer that the state should be very close to the separable vacuum state.

For comparison, Fig. 10 displays the corresponding mean number of photons, which, as expected, is indeed very low where there is no phase matching.

However, in order to understand the results of Fig. 9, we have to remind the reader that we are studying the degree of

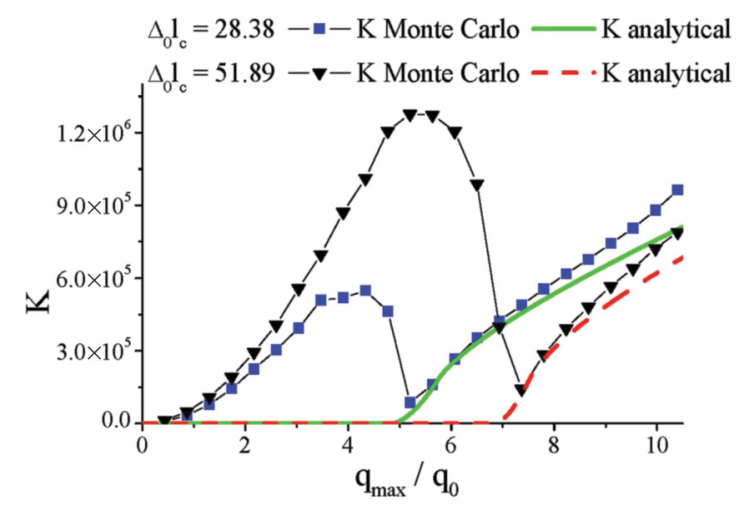

FIG. 9. (Color online) Schmidt number $\mathcal{K}$ in the noncollinear phase-matching case, as a function of the collected spatial bandwidth $q_{\max }$, normalized to the diffraction bandwidth $q_{0}$. Parameters are $\sigma_{p}=$ $600 \mu \mathrm{m}, \tau_{p}=1 \mathrm{ps}$.

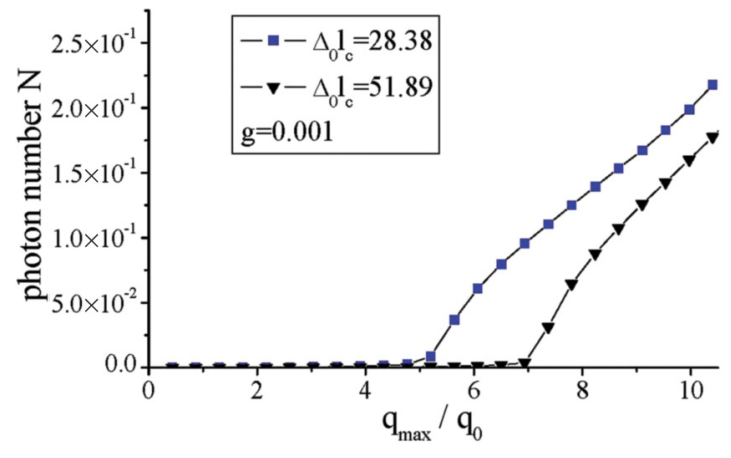

FIG. 10. (Color online) Number of photons $N$ in the noncollinear case as a function of $\bar{q}_{\max }$. Parameters are $\sigma_{p}=600 \mu \mathrm{m}, \tau_{p}=1 \mathrm{ps}$, $g=0.001$.

entanglement of the state (15), conditioned to the detection of a photon pair. This means that our calculation of the Schmidt number has lost track of the presence of a large vacuum contribution to the original PDC state, and the result has to be interpreted as photon pairs are emitted with very low probability; however, when a pair is detected, it is highly entangled.

The point to understand, therefore, is why the nonphasematched photon pairs are more entangled than the phasematched ones. The Schmidt number $\mathcal{K}=1 / \sum_{j} \lambda_{j}{ }^{2}$ provides an estimate of the number of significant eigenvalues of the Schmidt decomposition, i.e., the number of entangled eigenmodes that participate to the modal decomposition. In the region where no phase matching occurs (for $\bar{q}_{\max }<\sqrt{\Delta_{0} l_{c}}$ ), $\mathcal{K}$ can be, in practice, very large, since there is no mechanism for modal selection and all of the modes in the collected bandwidth participate equally to the PDC process even though with a very low occupation probability. By contrast, when increasing $\bar{q}_{\max }$ towards $\sqrt{\Delta_{0} l_{c}}$ and entering the phasematching region, a strongly reduced number of phase-matched spatiotemporal modes contribute to the Schmidt decomposition, i.e., the few which are close to satisfy the phase-matching condition $\bar{q}=\sqrt{\Delta_{0} l_{c}}, \Omega=0$. The number of significant eigenmodes is therefore reduced because phase matching operates a selection of the spatiotemporal modes that efficiently participate to the entanglement of the state. In other terms, the phase-matching mechanism favors only a small number of modes, thereby drastically reducing the dimensionality of the entangled state. By increasing $\bar{q}_{\max }$ above $\sqrt{\Delta_{0} l_{c}}$, the Schmidt number $\mathcal{K}$ again starts to increase, according to the geometrical interpretation of the Schmidt number, as being proportional again to the phase-matching volume.

\section{CONCLUSIONS}

In this work, we have calculated the Schmidt dimensionality of the two-photon state generated by PDC in the ultralow gain regime. Results have been produced with different degrees of approximation: Monte Carlo results have been obtained without relevant approximations, while in the limit of a broad pump (NPWPA), we could demonstrate a transparent geometrical interpretation of the Schmidt number, which can be seen as the number of entangled modes contained in the region where phase matching occurs. 
The same calculations have been performed in models restricted to the purely spatial or temporal degrees of freedom of biphotons. A remarkable result demonstrated is that when the collected spatiotemporal bandwidth is large enough, the Schmidt dimensionality of the full spatiotemporal state cannot be trivially reduced to the product of the Schmidt numbers characterizing the entanglement in lower dimensions. Therefore, obtaining the Schmidt number in the full 3D model is not a mere exercise of calculus: in order to correctly characterize the entanglement of twin photons, it is necessary to consider simultaneously their spatial and temporal degrees of freedom. This result is a clear consequence of the nonfactorability of the state in space and time, and mirrors the findings described in Refs. $[19,20]$, where the spatiotemporal correlation of the biphoton state was shown to have a nonfactorable $X$-shaped geometry.

The nonfactorability has been demonstrated in this work only in the NPWPA. The question is still open as to whether in the opposite limit, that is, for a very focused and short pump pulse, the Schmidt number keeps the nonfactorable character, and it is obviously linked to the more general question of whether the state appears to be factorable in space and time in these conditions. We remark, however, that reaching this limit is, in practice, very demanding because it requires that the pump Fourier profile at the exit face of the crystal be much broader than the width of the phase-matching function. For a few millimeter crystal, this implies a pump waist on the order of tens of microns, and a pulse duration as short as few tens of femtoseconds. While the first condition could be achieved, in principle, by strongly focusing at the end face of the crystal, the second is more demanding because of dispersion occurring inside the nonlinear material.

An intriguing finding is that in the absence of phase matching, where the probability of emission of photon pair is very low, the Schmidt dimensionality of the state is huge, and is actually larger than in the regions where phase matching occurs. This counterintuitive finding has been explained through the mode-selection mechanism performed by phase matching, which reduces the available number of spatiotemporal modes. However, in evaluating this result and its usefulness for applications, one has to remember that the Schmidt number analyzed here does not refer to the full PDC state, but to the state conditioned to the detection of a photon pair.

\section{ACKNOWLEDGMENTS}

We acknowledge support from the Fet Open programme of the EC within the Grant No. 221906 HIDEAS (High Dimensional Entangled Systems).

\section{APPENDIX A: PARTIAL DENSITY MATRIX}

In order to calculate the partial trace of the density matrix (18), it is enough to consider the vacuum state $|0\rangle_{2}$ of the "idler" subsystem 2, plus the continuous set of one-photon states,

$$
\left\{A_{2}^{\dagger}\left(\vec{w}_{2}\right)|0\rangle_{2}\right\} .
$$

It is convenient to write the density matrix (18) as

$$
\rho=\frac{1}{N} M^{\dagger}|0\rangle\langle 0| M,
$$

where $M$ is the operator that annihilates a photon pair in any spatiotemporal mode (weighted by $\psi$ ),

$$
M=\int d \vec{w}_{1} \int d \vec{w}_{2} \psi^{*}\left(\vec{w}_{1}, \vec{w}_{2}\right) A_{1}\left(\vec{w}_{1}\right) A_{2}\left(\vec{w}_{2}\right)
$$

We then have

$$
\begin{aligned}
\rho_{1}= & \operatorname{Tr}_{2}\{\rho\}=\frac{1}{N}\left\{{ }_{2}\left\langle 0\left|M^{\dagger}\right| 0\right\rangle_{2}|0\rangle_{1}{ }_{1}\left\langle 0\left|{ }_{2}\langle 0|M| 0\rangle_{2}+\int d \vec{w}_{3}{ }_{2}\left\langle 0\left|A_{2}\left(\vec{w}_{3}\right) M^{\dagger}\right| 0\right\rangle_{2}\right| 0\right\rangle_{1}{ }_{1}\langle 0|{ }_{2}\left\langle 0\left|M A_{2}^{\dagger}\left(\vec{w}_{3}\right)\right| 0\right\rangle_{2}\right\} \\
= & \frac{1}{N}\left\{\int d \vec{w}_{3} \int d \vec{w}_{1} \int d \vec{w}_{2} \int d \vec{w}_{1}^{\prime} \int d \vec{w}_{2}^{\prime} \psi\left(\vec{w}_{1}, \vec{w}_{2}\right) \psi^{*}\left(\vec{w}_{1}^{\prime}, \vec{w}_{2}^{\prime}\right)\right. \\
& \left.\times{ }_{2}\left\langle 0\left|A_{2}\left(\vec{w}_{3}\right) A_{2}^{\dagger}\left(\vec{w}_{2}\right)\right| 0\right\rangle_{2} A_{1}^{\dagger}\left(\vec{w}_{1}\right)|0\rangle_{1}{ }_{1}\langle 0| A_{1}\left(\vec{w}_{1}^{\prime}\right)_{2}\left\langle 0\left|A_{2}\left(\vec{w}_{2}\right) A_{2}^{\dagger}\left(\vec{w}_{3}\right)\right| 0\right\rangle_{2}\right\} \\
= & \frac{1}{N}\left\{\int d \vec{w}_{1} \int d \vec{w}_{2} \int d \vec{w}_{1}^{\prime} \psi\left(\vec{w}_{1}, \vec{w}_{2}\right) \psi^{*}\left(\vec{w}_{1}^{\prime}, \vec{w}_{2}\right) A_{1}^{\dagger}\left(\vec{w}_{1}\right)|0\rangle_{1}{ }_{1}\langle 0| A_{1}\left(\vec{w}_{1}^{\prime}\right)\right\} \\
= & \frac{1}{N}\left\{\int d \vec{w}_{1} \int d \vec{w}_{1}^{\prime} G\left(\vec{w}_{1}^{\prime}, \vec{w}_{1}\right) A_{1}^{\dagger}\left(\vec{w}_{1}\right)|0\rangle_{1}{ }_{1}\langle 0| A_{1}\left(\vec{w}_{1}^{\prime}\right)\right\},
\end{aligned}
$$

where, in passing from Eq. (A5) to Eq. (A6), we used the relation

$$
{ }_{2}\left\langle 0\left|A_{2}\left(\vec{w}_{2}\right) A_{2}^{\dagger}\left(\vec{w}_{2}^{\prime}\right)\right| 0\right\rangle_{2}=\delta\left(\vec{w}_{2}-\vec{w}_{2}^{\prime}\right),
$$

which comes directly from the commutation rules of bosonic operators. 


\section{APPENDIX B: DERIVATION OF FORMULA (31)}

We wish here to simplify the general formula (21) by exploiting the NPWPA introduced in Sec. IV [see Eq. (28)]. We rewrite formula (21) as

$$
\mathcal{K}=\frac{N^{\prime 2}}{B^{\prime}}
$$

with

$$
\begin{gathered}
B^{\prime}=\int d \vec{w}_{1} \int d \vec{w}_{2} \int d \vec{w}_{1}^{\prime} \int d \vec{w}_{2}^{\prime} \tilde{\mathcal{A}}_{p}\left(\vec{w}_{1}+\vec{w}_{2}\right) \tilde{\mathcal{A}}_{p}\left(\vec{w}_{1}^{\prime}+\vec{w}_{2}^{\prime}\right) \tilde{\mathcal{A}}_{p}{ }^{*}\left(\vec{w}_{1}^{\prime}+\vec{w}_{2}\right) \\
\times \tilde{\mathcal{A}}_{p}^{*}\left(\vec{w}_{1}^{\prime}+\vec{w}_{2}\right) \tilde{\mathcal{A}}_{p}^{*}\left(\vec{w}_{1}+\vec{w}_{2}^{\prime}\right) V\left(\vec{w}_{1}, \vec{w}_{2}\right) V\left(\vec{w}_{1}^{\prime}, \vec{w}_{2}^{\prime}\right) V^{*}\left(\vec{w}_{1}^{\prime}, \vec{w}_{2}\right) V^{*}\left(\vec{w}_{1}, \vec{w}_{2}^{\prime}\right), \\
N^{\prime}=\int d \vec{w}_{1} d \vec{w}_{2}\left|\tilde{\mathcal{A}}_{p}\left(\vec{w}_{1}+\vec{w}_{2}\right)\right|^{2}\left|V\left(\vec{w}_{1}, \vec{w}_{2}\right)\right|^{2},
\end{gathered}
$$

where we inserted the explicit expression (24) for the biphoton amplitude (without a constant coefficient). Here the function $V$ depends on phase matching and is given by Eq. (25), while $\tilde{\mathcal{A}}_{p}$ is the Fourier profile of the pump. We start by simplifying the integral $B^{\prime}$ in Eq. (B2) by introducing the change of variables,

$$
\left(\vec{w}_{1}, \vec{w}_{1}^{\prime} \vec{w}_{2}, \vec{w}_{2}^{\prime}\right) \rightarrow\left(\vec{w}_{1}, \vec{\delta}=\vec{w}_{1}-\vec{w}_{1}^{\prime}, \vec{w}_{p}=\vec{w}_{1}+\vec{w}_{2}, \vec{w}_{p}^{\prime}=\vec{w}_{1}^{\prime}+\vec{w}_{2}^{\prime}\right)
$$

With this change, $B^{\prime}$ becomes

$$
\begin{aligned}
B^{\prime}= & \int d \vec{w}_{p} d \vec{w}_{p}^{\prime} d \vec{\delta} d \vec{w}_{1} \tilde{\mathcal{A}}_{p}\left(\vec{w}_{p}\right) \tilde{\mathcal{A}}_{p}\left(\vec{w}_{p}^{\prime}\right) \tilde{\mathcal{A}}_{p}^{*}\left(\vec{w}_{p}-\vec{\delta}\right) \tilde{\mathcal{A}}_{p}^{*}\left(\vec{w}_{p}^{\prime}+\vec{\delta}\right) V\left(\vec{w}_{1},-\vec{w}_{1}+\vec{w}_{p}\right) \\
& \times V\left(\vec{w}_{1}-\vec{\delta},-\vec{w}_{1}+\vec{\delta}+\vec{w}_{p}^{\prime}\right) V^{*}\left(\vec{w}_{1}-\vec{\delta},-\vec{w}_{1}+\vec{w}_{p}\right) V^{*}\left(\vec{w}_{1},-\vec{w}_{1}+\vec{\delta}+\vec{w}_{p}^{\prime}\right)
\end{aligned}
$$

In this expression, the variables $\vec{w}_{p}$ and $\vec{w}_{p}^{\prime}$ die on the scale of the inverse of the pump waist and duration $2 / \sigma_{p}, 2 / \tau_{p}$. Because of the presence of the terms $\mathcal{A}_{p}^{*}\left(\vec{w}_{1}^{\prime}-\vec{w}_{1}+\underset{w_{p}}{p}\right)$ and $\mathcal{A}_{p}^{*}\left(\vec{w}_{1}-\vec{w}_{1}^{\prime}+\vec{w}_{p}^{\prime}\right)$, the variable $\delta=\vec{w}_{1}-\vec{w}_{1}^{\prime}$ also dies out on the same scale. We can then make use of the NPWP approximation, which amounts to substituting

$$
V\left(\vec{w}_{1},-\vec{w}_{1}+\vec{w}_{p}\right) \approx V\left(\vec{w}_{1}-\vec{\delta},-\vec{w}_{1}+\vec{\delta}+\vec{w}_{p}^{\prime}\right) \approx V\left(\vec{w}_{1},-\vec{w}_{1}\right):=V\left(\vec{w}_{1}\right)
$$

where we took into account that all of the pump variables $\vec{w}_{p}, \vec{\delta}, \vec{\delta}+\vec{w}_{p}^{\prime}$ die out on the fast scale of the inverse of the pump waist and duration, over which the function $V$ remains constant. Similarly,

$$
V^{*}\left(\vec{w}_{1}-\vec{\delta},-\vec{w}_{1}+\vec{w}_{p}\right) \approx V^{*}\left(\vec{w}_{1},-\vec{w}_{1}+\vec{\delta}+\vec{w}_{p}^{\prime}\right) \approx V^{*}\left(\vec{w}_{1},-\vec{w}_{1}\right):=V^{*}\left(\vec{w}_{1}\right) .
$$

Within the NPWP approximation, we hence obtain a new expression for $B^{\prime}$, which reads

$$
B^{\prime}=\int d \vec{w}_{p} d \vec{w}_{p}^{\prime} d \vec{\delta} \tilde{\mathcal{A}}_{p}\left(\vec{w}_{p}\right) \tilde{\mathcal{A}}_{p}\left(\vec{w}_{p}^{\prime}\right) \tilde{\mathcal{A}}_{p}{ }^{*}\left(\vec{w}_{p}-\vec{\delta}\right) \tilde{\mathcal{A}}_{p}{ }^{*}\left(\vec{w}_{p}^{\prime}+\vec{\delta}\right) \int d \vec{w}_{1}\left|V\left(\vec{w}_{1}\right)\right|^{4} .
$$

The integral over the pump variables can be further simplified by noting that it involves two convolutions:

$$
\int d \vec{w}_{p} \tilde{\mathcal{A}}_{p}\left(\vec{w}_{p}\right) \tilde{\mathcal{A}}_{p}^{*}\left(\vec{w}_{p} \pm \vec{\delta}\right)=\int d \vec{\xi}_{p}\left|\mathcal{A}_{p}\left(\vec{\xi}_{p}\right)\right|^{2} e^{ - \pm i \vec{\xi}_{p} \cdot \vec{\delta}}=(2 \pi)^{\frac{3}{2}}\left[\mathcal{F}\left(\left|\mathcal{A}_{p}\right|^{2}\right)\right]( \pm \vec{\delta}),
$$

where the symbol $\mathcal{F}(f)$ denotes the Fourier transform of the function $f$. By also performing the integration over $\vec{\delta}$, we obtain

$$
\int d \vec{\delta}\left[\mathcal{F}\left(\left|\mathcal{A}_{p}\right|^{2}\right)\right](\vec{\delta})\left[\mathcal{F}\left(\left|\mathcal{A}_{p}\right|^{2}\right)\right](-\vec{\delta})=\int d \vec{\xi}_{p}\left|\mathcal{A}_{p}\left(\vec{\xi}_{p}\right)\right|^{4},
$$

where we used the Plancherel theorem $\int d^{3} \vec{w}|\tilde{f}(\vec{w})|^{2}=\int d^{3} \xi|f(\xi)|^{2}$. This leads to

$$
B^{\prime}=(2 \pi)^{3}\left[\int d \vec{\xi}_{p}\left|\mathcal{A}_{p}\left(\xi_{p}\right)\right|^{4}\right]\left[\int d \vec{w}\left|V_{p w}(\vec{w})\right|^{4}\right] .
$$

In order to complete the Schmidt-number calculation, we also have to evaluate $N^{\prime}$, proportional to the mean photon number. With the usual change of variables, $\left(\vec{w}_{1}, \vec{w}_{2}\right) \rightarrow\left(\vec{w}_{p}=\vec{w}_{1}+\vec{w}_{2}, \vec{w}_{1}\right)$, Eq. (B3) becomes

$$
N^{\prime}=\int d \vec{w}_{p}\left|\tilde{\mathcal{A}}_{p}\left(\vec{w}_{p}\right)\right|^{2} \int d \vec{w}_{1}\left|V\left(\vec{w}_{1},-\vec{w}_{1}+\vec{w}_{p}\right)\right|^{2} .
$$

In the NPWP limit [see Eq. (28)], we get

$$
N^{\prime}=\int d \vec{w}_{p}\left|\tilde{\mathcal{A}}_{p}\left(\vec{w}_{p}\right)\right|^{2} \int d \vec{w}|V(\vec{w})|^{2},
$$


which, using the Plancherel theorem in the first integral, can be also written as

$$
N^{\prime}=\int d \vec{\xi}_{p}\left|\tilde{\mathcal{A}}_{p}\left(\vec{\xi}_{p}\right)\right|^{2} \int d \vec{w}|V(\vec{w})|^{2}
$$

In the NPWP limit, the Schmidt number takes therefore the simplified form

$$
\mathcal{K}=\frac{N^{2}}{B}=\frac{\left[\int d \vec{\xi}_{p}\left|\mathcal{A}_{p}\left(\vec{\xi}_{p}\right)\right|^{2}\right]^{2}}{\int d \vec{\xi}_{p}\left|\mathcal{A}_{p}\left(\vec{\xi}_{p}\right)\right|^{4}} \frac{\left[\int d \vec{w}|V(\vec{w})|^{2}\right]^{2}}{(2 \pi)^{3} \int d \vec{w}|V(\vec{w})|^{4}},
$$

where the integrals now factorize into the pump and signal degrees of freedom.

[1] See, e.g., the website of the project HIDEAS at http://hideas.dfm.uninsubria.it/, and the papers listed there.

[2] A. Ekert and P. L. Knight, Am. J. Phys. 63, 415 (1995).

[3] S. Parker, S. Bose, and M. B. Plenio, Phys. Rev. A 61, 032305 (2000).

[4] C. K. Law, I. A. Walmsley, and J. H. Eberly, Phys. Rev. Lett. 84, 5304 (2000).

[5] Y. M. Mikhailova, P. A. Volkov, and M. V. Fedorov, Phys. Rev. A 78, 062327 (2008).

[6] G. Patera, G. De Valcarcel, N. Treps, and C. Fabre, Eur. Phys. J. D 56, 123 (2010).

[7] O. Pinel, Pu Jian, R. M. deAraujo, Jinxia Feng, B. Chalopin, C. Fabre, and N. Treps, Phys. Rev. Lett. 108, 083601 (2012).

[8] Malte Avenhaus, Maria V. Chekhova, Leonid A. Krivitsky, Gerd Leuchs, and Christine Silberhorn, Phys. Rev. A 79, 043836 (2009).

[9] C. K. Law and J. H. Eberly, Phys. Rev. Lett. 92, 127903 (2004).

[10] M. P. van Exter, A. Aiello, S. S. R. Oemrawsingh, G. Nienhuis, and J. P. Woerdman, Phys. Rev. A 74, 012309 (2006).

[11] H. Di Lorenzo Pires, C. H. Monken, and M. P. van Exter, Phys. Rev. A 80, 022307 (2009).

[12] H. Di Lorenzo Pires, H. C. B. Florijn, and M. P. van Exter, Phys. Rev. Lett. 104, 020505 (2010).

[13] S. S. Straupe, D. P. Ivanov, A. A. Kalinkin, I. B. Bobrov, and S. P. Kulik, Phys. Rev. A 83, 060302(R) (2011).

[14] J. P. Torres, A. Alexandrescu, and Lluis Torner, Phys. Rev. A 68, 050301(R) (2003).

[15] Gabriel Molina-Terriza, Juan P. Torres, and Lluis Torner, Nature Phys. 3, 305 (2007).
[16] Sonja Franke-Arnold, Les Allen, and Miles Padgett, Laser Photon. Rev. 2, 299 (2008).

[17] A. C. Dada, J. Leach, G. S. Buller, M. J. Padgett, and E. Andersson, Nature Phys. 7, 677 (2011).

[18] See, e.g., A. Gatti, E. Brambilla, and L. Lugiato, Quantum Imag. Prog. Opt. 51, 251 (2008).

[19] A. Gatti, E. Brambilla, L. Caspani, O. Jedrkiewicz, and L. A. Lugiato, Phys. Rev. Lett. 102, 223601 (2009).

[20] L. Caspani, E. Brambilla, and A. Gatti, Phys. Rev. A 81, 033808 (2010).

[21] E. Brambilla, L. Caspani, L. A. Lugiato, and A. Gatti, Phys. Rev. A 82, 013835 (2010).

[22] O. Jedrkiewicz, J.-L. Blanchet, E. Brambilla, P. Di Trapani, and A. Gatti, Phys. Rev. Lett. 108, 253904 (2012).

[23] Enrico Brambilla, Ottavia Jedrkiewicz, Luigi Alberto Lugiato, and Alessandra Gatti, Phys. Rev. A 85, 063834 (2012).

[24] O. Jedrkiewicz, A. Gatti, E. Brambilla, and P. Di Trapani, arXiv:1207.6934 (2012) [Phys. Rev. Lett. (to be published)].

[25] Clara I. Osorio, Alejandra Valencia, and Juan P. Torres, New J. Phys. 10, 113012 (2008).

[26] D. B. Horoshko, G. Patera, A. Gatti, and M. I. Kolobov, Eur. Phys. J. D 66, 239 (2012).

[27] A. Gatti, R. Zambrini, M. San Miguel, and L. A. Lugiato, Phys. Rev. A 68, 053807 (2003).

[28] J. M. Hammersley and D. C. Handscomb, Monte Carlo Methods (Chapman and Hall, London, 1964).

[29] V. G. Dmitriev, G. G. Gurzadyan, and D. N. Nikogosyan, Handbook of Nonlinear Optical Crystals, Springer Series in Optical Sciences, edited by A. E. Siegman (Springer-Verlag, Berlin, 1991); N. Boeuf et al., Opt. Eng. 39, 1016 (2000). 